\title{
Detection and identification of peroxiredoxin 3 as a biomarker in hepatocellular carcinoma by a proteomic approach
}

\author{
BINGBING QIAO $^{1}$, JIAJIA WANG ${ }^{2}$, JIANFEI XIE ${ }^{1}$, YING NIU $^{1}$, SHAOJUN YE $^{1}$, QIQUAN WAN ${ }^{1}$ and QIFA YE $^{1,2}$ \\ ${ }^{1}$ Department of Surgery, The Third Xiangya Hospital, Central South University, Changsha, Hunan 410013; ${ }^{2}$ Hepatobiliary \\ Disease Institution, Zhongnan Hospital of Wuhan University, Wuhan University, Wuhan, Hubei 430071, P.R. China
}

Received December 15, 2011; Accepted January 27, 2012

DOI: $10.3892 / \mathrm{ijmm} .2012 .916$

\begin{abstract}
Hepatocellular carcinoma (HCC) is one of the most common malignancies worldwide with poor prognosis due to resistance to conventional chemotherapy and limited efficacy of radiotherapy. Thus, alternative therapeutic strategies need to be established. In order to search for a useful biomarker to improve its efficacy, we conducted a two-dimensional gel electrophoresis and MALDI-TOF MS-based comparative proteomic analysis to profile the differentially expressed proteins between HCC tumor tissues with histological evidence and the adjacent non-tumor tissues. Twenty-two out of 43 dysregulated proteins were identified, including 15 upregulated proteins, and 7 downregulated proteins (over 2 -fold, $\mathrm{P}<0.01$ ). The expression of peroxiredoxin 3 (PRDX3) at the mRNA and protein levels was confirmed by RT-PCR and western blotting in HCC cell lines, and HCC samples, and further analysed by immunohistochemistry in HCC samples of different clinical pathological stages. The results indicated that overexpression of PRDX3 was associated with 94.9\% HCC, and correlated with poor differentiation $(\mathrm{P}<0.05)$, which suggest that PRDX3 has substantial clinical impact on the progression of hepatocarcinoma, and may be a potential therapeutic target for HCC.
\end{abstract}

\section{Introduction}

Hepatocellular carcinoma (HCC) is one of the most common malignancies worldwide with poor prognosis due to limited efficacy of radiotherapy and resistance to traditional chemotherapy (1). Chronic infection with hepatitis $\mathrm{B}$ or $\mathrm{C}$ virus has been thought as the major etiologic factor, accounting for about $80 \%$ of all HCC cases. Due to a high frequency of metastasis/

Correspondence to: Professor Qifa Ye, Department of Surgery, The Third Xiangya Hospital, Central South University, Changsha, Hunan 410013, P.R. China

E-mail: yqf_china@163.com

Key words: hepatocellular carcinoma, peroxiredoxin 3, oxidative stress, biomarker recurrence, 5 year survival rate is less than $30 \%$, and about one-third of the HCC patients relapse within 1 year after curative surgery $(2,3)$. Many potentials for clinical therapy in HCC have been identified, however, most of them were not particularly efficient in treating HCC. Consequently, there is an urgent need to screen for novel biomarkers and therapeutic targets with high sensitivity, specificity and operability.

Proteomics approaches are promising tools for the discovery of new tumor-associated biomarkers and therapeutic drug targets (4). In addition to the better genetic and epigenetic alterations, there are other factors related to molecular changes in translation, post-translational modification, and intracellular mislocalization involved in tumor initiation and growth, and these factors cannot be detected either by measuring the amount of RNA or by detecting nucleotide sequence variation (5). By examining the expression abundance and post-translational modification of hundreds of proteins, and further comparative analysis of proteomes between different samples, proteomics can directly reveal the genetic information of many types of cancer. For comparative proteomics studies, two dimensional gel electrophoresis (2-DE) followed by mass spectrometry analysis is the core technology used in finding the differentially displayed proteins and identifying the novel biomarkers (6). Biomarkers associated with the progression of cancer, such as glutathione S-transferase pi 1 (GSTP1), des- $\gamma$-carboxyprothrombin (DCP), and heat shock protein 27 (HSP 27) have been identified (7-9), however, their application in clinical therapy is limited. In our present study, 22 differential proteins with a variety of biological functions were identified by Matrix-Assisted Laser Desorption/ Ionization Time of Flight Mass Spectrometry (MALDI-TOF MS). Among these, thioredoxin-dependent peroxide reductase, mitochondrial peroxiredoxin 3 (PRDX3) is of great interest due to its high expression in HCC.

Several investigations demonstrated that HCC was closely correlated to oxidative stress (10). Cancer cells usually alter their key mitochondrial regulators of cell death and their mitochondrial structure and function is different from that of normal cells. This enables them to use antioxidant systems to maintain the redox balance under high oxidative stresses (11). Classical antioxidant genes related to cancer are the superoxide dismutase (SOD), catalase, GST, glutathione peroxidase, peroxiredoxins and thioredoxin (Trx) (12-15). Peroxiredoxins play important roles in peroxide detoxification, and belong 
to a family of thiol-specific antioxidant enzymes catalyzing the reduction of hydrogen peroxide $\left(\mathrm{H}_{2} \mathrm{O}_{2}\right)$ in the presence of thioredoxin (16). The 6 Prx members in humans have been shown to participate in regulating cell signaling, influencing cell differentiation and proliferation, and immune responses, and protecting free radical sensitive protein from oxidative damage (17). Among them, PRDX3 specifically localizes in the mitochondria, as a c-Myc target gene required for mitochondrial homeostasis and neoplastic transformation (18). It is believed to play important roles in the regulation of cellular redox status by serving as a primary line of defense against $\mathrm{H}_{2} \mathrm{O}_{2}$ produced during respiration, and protects cells from $\mathrm{H}_{2} \mathrm{O}_{2}$-induced apoptosis (19). PRDX3 has been shown to be overexpressed in mesothelioma, hepatocellular carcinoma, breast cancer and ovarian cancer (20-23), which suggest that PRDX3 may be involved in tumorigenesis and progression.

In the present study, we analyzed the differential proteome profiles between HCC and adjacent non-tumor tissues to identify the potential molecules as tumor therapeutic targets. We identified 15 upregulated and 7 downregulated proteins with various of biological functions by MALDI-TOF/TOF tandem mass spectrometry. PRDX3, which was significantly upregulated in HCC tissues compared with the adjacent non-tumor tissues, was chosen for validation and analysis. Clinical correlation of PRDX3 with different clinicopathological features in HCC was also evaluated. The data presented in this study demonstrates that overexpression of PRDX3 is closely associated with the progression of hepatocarcinoma, and targeting PRDX3 may inhibit malignant transformation and progression. These findings have potential therapeutic implications.

\section{Materials and methods}

Cell lines and clinical specimens. Human hepatoma HepG2, Hep3B, QGY-7703, HuH7 cell lines and the normal liver cell line QSG-7701 were obtained from the American Type Culture Collection (ATCC). All cell lines were maintained in Dulbecco's modified Eagle's medium (DMEM) (Gibco, USA) containing $10 \%$ fetal bovine serum (Gibco), $100 \mathrm{U} / \mathrm{ml}$ of penicillin, and $100 \mu \mathrm{g} / \mathrm{ml}$ of streptomycin in a humidified incubator at $37^{\circ} \mathrm{C}$ with $5 \% \mathrm{CO}_{2}$.

For proteomics analysis, 27 pairs of human frozen $\mathrm{HCC}$ histologically-confirmed tissues and the adjacent non-tumor tissues were obtained from the Department of Surgery, Third Xiangya Hospital of Central South University. Informed consent was obtained from each patient. Tissues were snapfrozen in liquid nitrogen after surgical resection and stored at $-80^{\circ} \mathrm{C}$ until use. For the validation studies, $119 \mathrm{HCC}$ tissue samples and 36 adjacent non-tumor/normal liver specimens were obtained between the years 2009 and 2011. A summary of clinical information for these patients is shown in Table II.

Protein extraction and 2-DE proteomics profiling. Proteins from tumor and matched non-tumor tissues were extracted using the ReadyPrep Sequential Extraction kit (Bio-Rad, USA) according to the manufacturer's protocol. Samples were cleaned up using a PlusOne 2-D Clean-up kit (GE Healthcare, USA), and dissolved with sample buffer [7 $\mathrm{M}$ urea, $2 \mathrm{M}$ thiourea, 4\% CHAPS, $1 \%$ Mega-10, $0.5 \%$ Triton X-100, 1\% IPG buffer ( $\mathrm{pH} 4-7)$ ], $50 \mathrm{mM}$ dithiothreitol and $2 \mathrm{mM}$ tributyl- phosphine). Proteins of $150 \mu \mathrm{g}$ from tumor and non-tumor tissues were separately mixed with rehydration buffer [7 $\mathrm{M}$ urea, $2 \mathrm{M}$ thiourea, 4\% CHAPS, 0.5\% IPG buffer ( $\mathrm{pH} 4-7$ )] followed by rehydration for $12 \mathrm{~h}$ at $30 \mathrm{~V}$ using the Immobiline IPG DryStrips (18 cm, pH 4-7) (GE Healthcare), and then were isoelectrically focused at $500 \mathrm{~V}$ for $1 \mathrm{~h}, 1,000 \mathrm{~V}$ for $1 \mathrm{~h}$, $3,000 \mathrm{~V}$ for $1 \mathrm{~h}$ and $8,000 \mathrm{~V}$ for $9.5 \mathrm{~h}$ on an Ettan IPGphor Electrophoresis system (GE Healthcare). After equilibration for $2 \times 15 \mathrm{~min}$ in equilibration buffer, IPG strips were applied for $12 \%$ sodium dodecyl sulfate-polyacrylamide gel electrophoresis (SDS-PAGE) using a PROTEAN ${ }^{\circledR}$ II xi Cell system (Bio-Rad). Each sample was run in triplicate.

Image acquisition and analysis. The gels were stained using Coomassie Brilliant Blue R-350 (Amersham Biosciences) according to the manufacturer's protocol. The protein spots were detected, quantified, and matched using PD-Quest 2D analysis software (Bio-Rad). Differences in the expression levels between paired samples were analyzed by the Student's t-test. Protein spots showing at least 2-fold changes in intensity were picked for trypsin digestion and MALDI-TOF MS analysis.

In-gel tryptic digestion and protein identification by mass spectrometry. The protein spots were excised from the gel and subjected to destaining, washing, and trypsin digestion (Promega) at $37^{\circ} \mathrm{C}$ overnight. The tryptic peptides were extracted from the gel and dried by centrifugal lyophilization. Peptide mixtures were redissolved in $0.5 \%$ TFA and analyzed by an ABI 4700 proteomics analyzer (Applied Biosystems, USA). Protein identification was performed using the Mascot software (Matrix Science, UK) and all tandem mass spectra were searched against the Swiss-Prot protein database. The search parameters included potential residue mass modification for carbamidomethylation and oxidation, one missed trypsin cleavage, and fragment ion tolerance and peptide tolerance of $\pm 0.2 \mathrm{Da}$. Up to one missed tryptic cleavage was considered for all tryptic-mass searches. Protein scores $>56$ were considered to be significant $(\mathrm{P}<0.05)$. Redundancy of proteins that appeared in the database under different names and accession numbers was eliminated. If more than one protein was identified in one spot the single protein member with the highest protein score was singled out from the multiprotein family.

Reverse transcriptase-polymerase chain reaction ( $R T-P C R)$. Total-RNA was extracted from frozen liver tissues or cell lines HepG2, QSG-7701 using TRIzol reagent following the manufacturer's instructions (Invitrogen, USA). RT-PCR was used for the analysis of human PRDX3 mRNA with glyceraldehyde 3-phosphate dehydrogenase (GAPDH) as an internal control. The following primer sequences were used to detect PRDX3 transcripts: forward primer, 5'-TGGTTCCAGTCAAGCAA AAT-3' and reverse primer, 5'-AACCTTCTAACAGCACACC GT-3'. The GAPDH primers were as follows: forward, 5'-TG ATGACATCAAGAAGGTGGTGAAG-3' and reverse, 5'-TC CTTGGAGGCCATGTGGGCCAT-3'. Reactions were performed in accordance with the standard protocol. PCR was performed initially by reverse transcription at $42^{\circ} \mathrm{C}$ for $60 \mathrm{~min}$ and denaturation at $95^{\circ} \mathrm{C}$ for $2 \mathrm{~min}$, then amplification for 
35 cycles at $94^{\circ} \mathrm{C}$ for $30 \mathrm{sec}$, annealing at $55^{\circ} \mathrm{C}$ for $30 \mathrm{sec}$, and extension at $72^{\circ} \mathrm{C}$ for $30 \mathrm{sec}$, followed by a terminal elongation step at $72^{\circ} \mathrm{C}$ for $10 \mathrm{~min}$. PCR products were analyzed by electrophoresis through $1.5 \%$ agarose and visualized with ethidium bromide. Experiments were performed in triplicate.

Western blot analysis. Total proteins of $50 \mu \mathrm{g}$ from frozen liver tissues or cell lines HepG2, Hep3B, QGY-7703, HuH7, and QSG-7701 were separated by 12\% SDS-PAGE and transferred onto poly-vinylidene difluoride (PVDF) membranes (Amersham Biosciences). The detection of proteins was carried out by incubating the membranes with anti-PRDX3 monoclonal antibody (diluted 1:1,000, Abcam) or mouse anti-human $\beta$-actin antibody (Santa Cruz Biotechnology, Inc., USA) at 1:1,000 dilution overnight at $4^{\circ} \mathrm{C}$ and followed by peroxidase-conjugated secondary antibody (Invitrogen). After substrate development by ECL chemiluminescence (GE Healthcare), signal intensity was quantitatively analyzed using the Quantity One software (Bio-Rad).

Immunohistochemistry. Consecutive formalin-fixed and paraffin-embedded tissue sections (3-5 $\mu \mathrm{m}$ ) for immunohistochemistry (IHC) analysis were dewaxed and rehydrated. Antigen retrieval was performed by pretreatment of the slides in citrate buffer ( $\mathrm{pH}$ 6.0) in a microwave oven for $15 \mathrm{~min}$. Endogenous peroxidase activity was quenched by incubating the slides in methanol containing $0.6 \% \mathrm{H}_{2} \mathrm{O}_{2}$ at room temperature. For PRDX3-specific staining, the sections were blocked by normal goat serum for $1 \mathrm{~h}$ at $37^{\circ} \mathrm{C}$, and subsequently, incubated with anti-human PRDX3 antibody (diluted 1:100, Abcam) at $4^{\circ} \mathrm{C}$ overnight, and rinsed with PBS containing $0.1 \%$ bovine serum albumin. Incubation with horseradish peroxidase-linked goat anti-mouse antibodies at $37^{\circ} \mathrm{C}$ for $30 \mathrm{~min}$ was followed by reaction with 3,3'-diaminobenzidine substrate solution and counterstaining with Mayer's hematoxylin.

The immunohistochemical staining was assessed by calculating the percentage of positive hepatocytes and the immunostaining intensity. Immunostaining intensity (i) was classified as lack of staining, 0 ; mild staining, 1 ; moderate staining, 2; and strong staining, 3 . The percentage of stainingpositive stroma (ii) was semiquantitatively divided into five grades: $<5 \%, 0 ; 6-25 \%, 1 ; 26-50 \%, 2 ; 51-75 \%, 3$; and $>75 \%, 4$. The score for each section was measured as (i) $\mathrm{x}$ (ii), and the result was defined as negative $(-, 0)$, weakly positive $(+, 1-3)$, positive (++, 4-7), and strongly positive (+++, 8-12). A minimum of five fields for each section were evaluated. Slides were examined separately by two independent pathologists without any prior knowledge of each patient's clinical and pathological parameters. Any discrepancy between the two evaluators was resolved by reevaluation and careful discussion until agreement was reached.

Statistical analysis. Statistical analyses were performed by SPSS 13.0 software. All data are expressed as mean \pm SD . Comparisons among multiple groups were performed by one-way ANOVA, or LSD t-test. Difference between two groups were analyzed by the LSD t-test. Relevance analysis of ordinal data was analyzed by the cross-tabulation $\chi^{2}$ test. $\mathrm{P}$-value $<0.05$ was considered statistically significant.

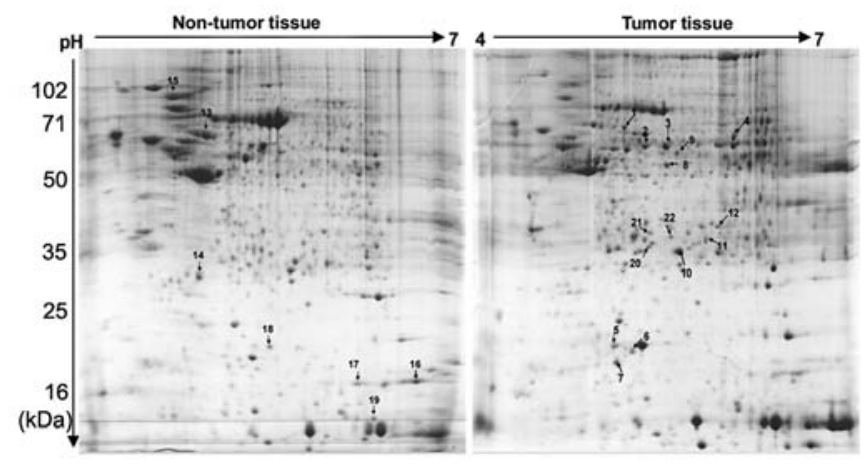

Figure 1. Differential proteomics analysis of HCC and the adjacent non-tumor tissues using 2-DE gels. Total protein lysates were separated by isoelectric focusing (IEF) using IPG strips (18 cm, pH 4-7) in the first dimension followed by $12 \%$ SDS-PAGE in the second dimension. Coomassie-stained gels were analyzed using the PD-Quest 2D analysis software. Totally, 22 out of 43 differentially expressed spots were identified by MS/MS analysis (marked with arrows and numbers).

\section{Results}

Proteomic profiling of differentially expressed proteins between tumor and non-tumor tissues. The 2-DE-based proteomic profiling approach was employed in triplicate to analyze the differential expressed proteins in 27 pairs of tumor and the matched non-tumor liver tissues resected from HCC patients who received curative hepatic surgery. With the PD-Quest 2D analysis software, statistical analysis of resultant 2-D gels revealed that more than 846 spots were detected in each Coomassie stained gel, and 22 out of 43 protein spots differentially expressed between HCC and adjacent non-tumor tissues were identified by MS/MS analysis ( $>2.0$-fold increase or decrease, $\mathrm{P}<0.05$ ). Their positions on the 2-DE map are annotated in Fig. 1.

Mass spectrum identification and functional classification of differentially expressed proteins. After MALDI-TOF/ TOF tandem mass spectrometry analysis and Swiss-Prot database search, 22 proteins identified from 43 spots (Table I). Of these proteins, 15 were upregulated, whereas the other 7 were downregulated in HCC. To understand the biological relation of the identified molecules with the progression of HCC, the identified 22 proteins were classified according to their biological functions with the tools on www.uniprot. org (Table I). These proteins were functionally involved in diverse biological processes, including metabolism (9/22, $40.9 \%)$, anti-apoptosis $(4 / 22,18.2 \%)$, signal transduction $(7 / 22$, $31.8 \%)$, redox regulation $(5 / 22,22.7 \%)$, structural protein $(3 / 22,13,6 \%)$, detoxification $(2 / 22,9.1 \%)$, chaperone $(3 / 22$, $13.6 \%)$, proteolysis $(1 / 22,4.5 \%)$, DNA synthesis and cell differentiation $(2 / 22,9.1 \%)$. Of the 22 proteins, $13(59.1 \%)$ were cytoplasmic, 2 (9.1\%) were cytoplasmic and nuclear, 2 (9.1\%) were membranous, 4 (18.2\%) were mitochondrial, and 1 (4.5\%) was a secreted protein (Table I).

Most of these identified proteins had been reported elsewhere and their role in the progression of HCC is clearly understood. PRDX3 plays important roles in the regulation of cellular redox balance, and may be involved in the progression 


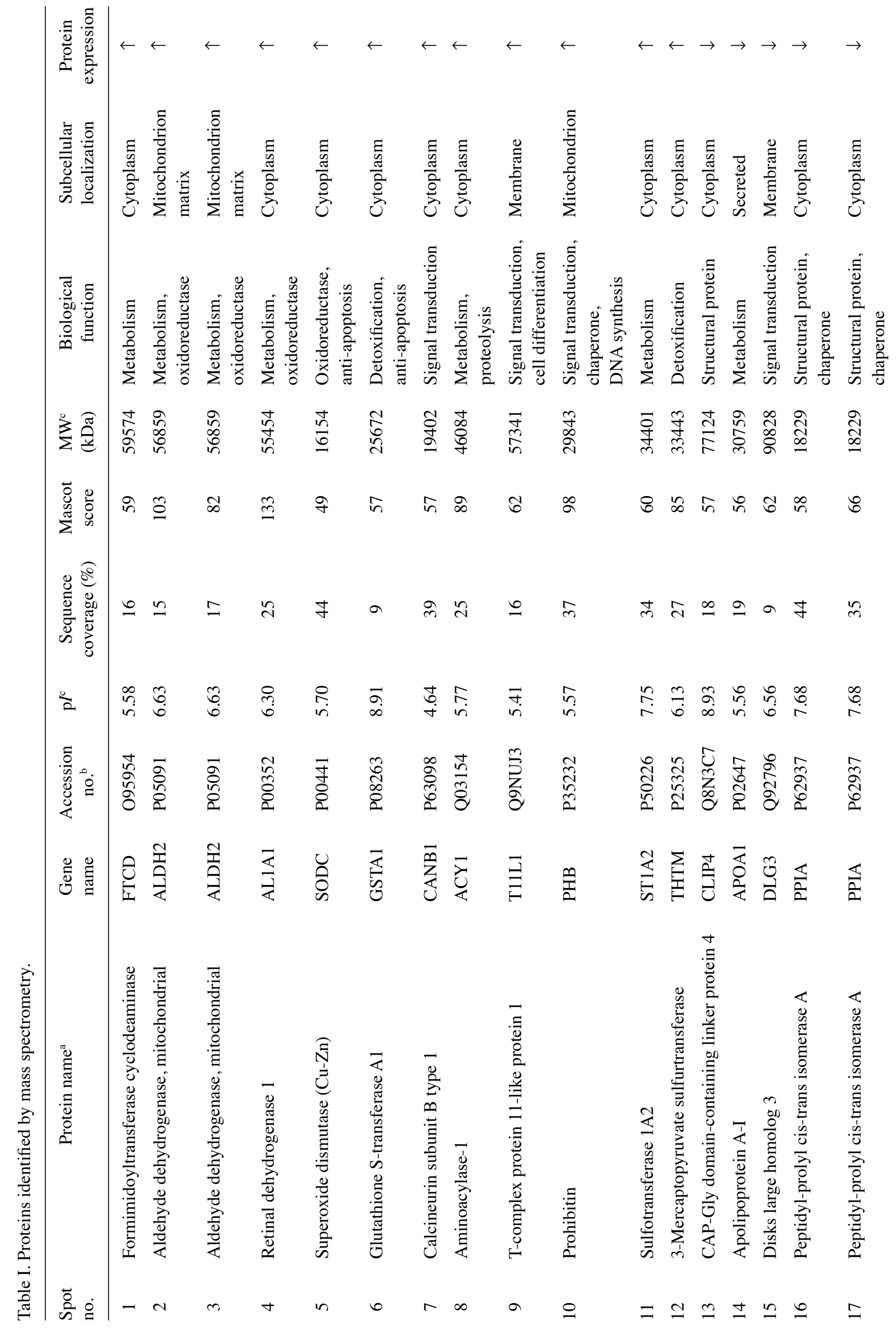




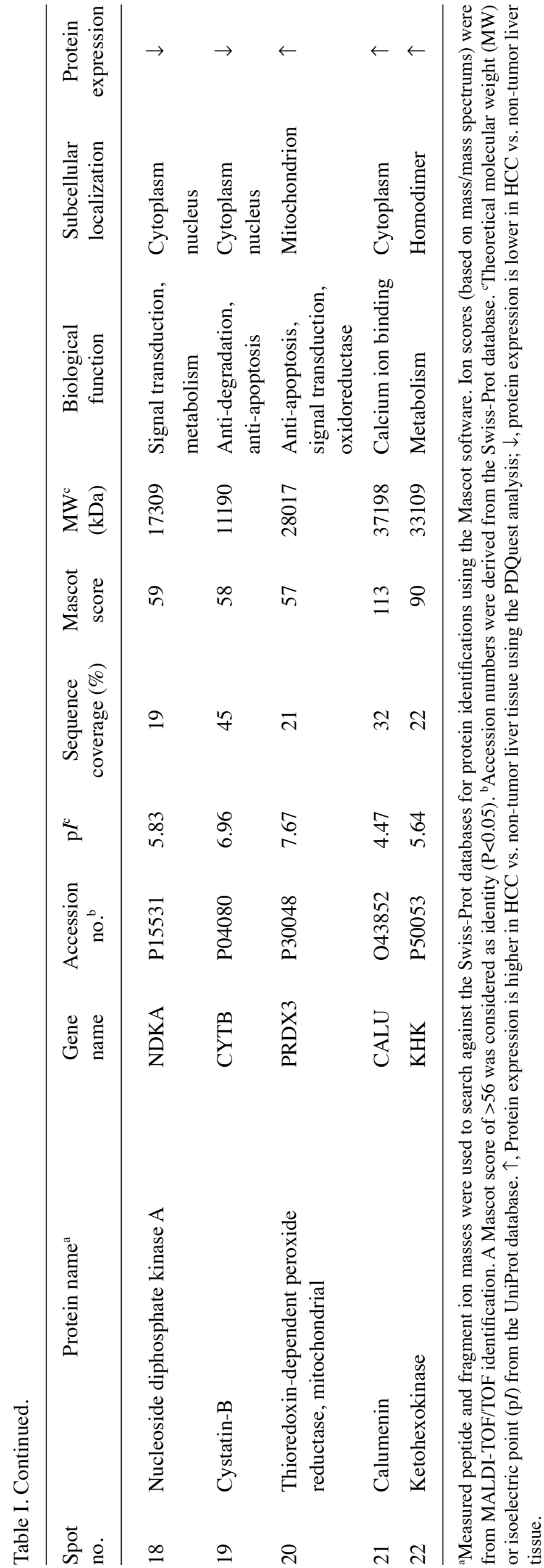

of HCC, however, there are few reports on the relationship between PRDX3 and the progression of HCC. Thus, PRDX3 became the subsequent focus of this study. Its representative 2-DE spot images with the MS/MS spectrum are shown in Fig. 2A. The predicted molecular mass/pI value for PRDX3 was $28.02 \mathrm{kDa} / 7.67$, fitting well to the position of the corresponding spot (spot 20) on the 2-DE gel. MS/MS analysis showed that PRDX3 was identified with a Mascot score of 57 and $21 \%$ sequence coverage (Fig. $2 \mathrm{~B}$ ).

Validation of PRDX3 by semiquantitative RT-PCR and western blot analysis. As the expression of PRDX3 in HCC had not been previously identified, we further confirmed its differential expression by RT-PCR and western blotting in 40 pairs of HCC and the adjacent non-tumor tissues, or in the hepatoma cell lines HepG2, Hep3B, QGY-7703, HuH7 and normal liver cell line QSG-7701. RT-PCR analysis showed that relative expression abundance of PRDX3 at the mRNA level in $\mathrm{HCC}$ was $0.79 \pm 0.14$, which was significantly higher than that in non-tumor tissues $(0.15 \pm 0.05)(\mathrm{P}<0.05)$ (Fig. 3B). The relative abundance of PRDX3 mRNA in HepG2, Hep3B, QGY-7703, HuH7 and QSG-7701 were 0.28 $\pm 0.05,0.40 \pm 0.06$, $0.32 \pm 0.04,0.30 \pm 0.02$ and $0.10 \pm 0.04$, respectively $(\mathrm{P}<0.05$, vs. QSG-7701 group) (Fig. 3E and F).

Western blot analysis was performed using an anti-PRDX3 antibody. The overall expression levels in the tumor tissues $(0.82 \pm 0.13)$ were higher than those in the matched non-tumor tissues (0.52 \pm 0.09$)$ (Fig. 3D), which was consistent with the results of the cell lines. These results confirmed that higher levels of PRDX3 were expressed in HCC or hepatoma cell lines compared with non-tumor tissues or normal liver cell line at both the mRNA and protein levels (Fig. 3). Also, expression levels of PRDX3 are shown to be case-dependent and higher mRNA $(0.83 \pm 0.24)$ and protein levels $(0.86 \pm 0.21)$ of PRDX3 were found in HCC with distant metastasis (data not shown). These results showed that the increasing trend paralleled the increasing severity of liver injury.

Overexpression of PRDX3 is correlated with poor differentiation of HCC. To investigate the oncogenic properties of PRDX3 in the progression of HCC, paraffin-embedded tissues were stained using anti-human PRDX3 antibody. HCC tissues $(n=119)$ at different clinicopathologic stages and adjacent non-tumor/normal liver tissues $(n=36)$ were prepared for immunohistochemical analysis. Of the 119 HCC samples, 27 were well differentiated, 74 were moderately differentiated, and 18 were poorly differentiated; 17 were in stage I, 56 were in stage II, 42 were in stage III, and 4 were in stage IV according to histodifferentiation criteria/TNM staging (Table II).

Staining for PRDX3 was mainly detected in the HCC cells (Fig. 4). In 36 adjacent non-tumor/normal specimens, no staining and weakly positive staining were detected in 17 and $83 \%$ of the samples, respectively. By contrast, in tumor tissues, weakly-positive staining was observed in 5.0\% (6/119), moderately positive staining in about $61.3 \%(73 / 119)$ and the strong positive staining in $33.7 \%$ (40/119) (Table II). The staining intensity and the number of positively stained cells were markedly different between non-tumor tissues and hepatoma tissues $(\mathrm{P}<0.01)$ (Fig. 4). Overexpression of PRDX3 was more likely to occur in poorly-differentiated tissues $(\mathrm{P}<0.05)$. 

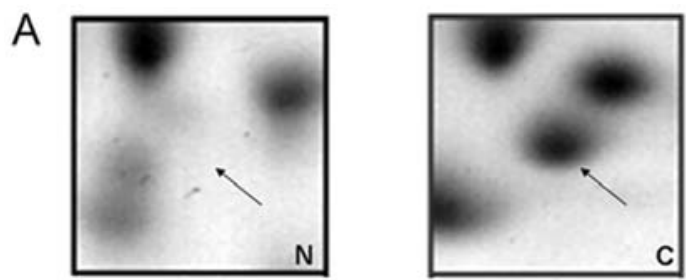

B

Voyager Spec $\# 1[B P=1462.9,2056]$

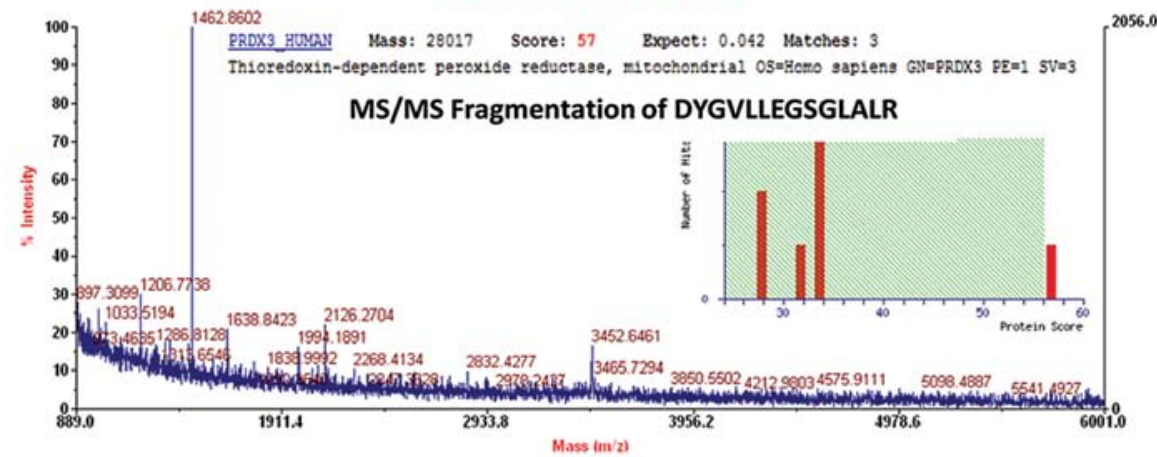

Figure 2. Identification of PRDX3 by MALDI-TOF tandem mass spectrometry. (A) 2-DE gel images of PRDX3 in HCC tissues and non-tumor controls. PRDX3 was upregulated more than 3-fold in HCC tissues when compared with the non-tumor controls. (B) Acquired spectra were searched against a Mascot Search engine based on the Swiss-Prot protein database. MS/MS spectrum of the precursor ion with $\mathrm{m} / \mathrm{z} 1462.9$ for peptide 171DYGVLLEGSGLALR184 with corresponding peak values was identified as PRDX3. Inserted figure is the Swiss-Prot protein score from the Swiss-Prot database. N, non-tumor tissues; C, cancer tissues.

Table II. Clinical characteristics of HCC samples from 119 patients.

PRDX3 expression in IHC

\begin{tabular}{|c|c|c|c|c|c|c|}
\hline \multirow{2}{*}{ Clinical features } & \multirow[b]{2}{*}{$\mathrm{N}$} & & & & & \\
\hline & & - & + & ++ & +++ & P-value \\
\hline Gender & & & & & & $P>0.05$ \\
\hline Male & 108 & 0 & 5 & 67 & 36 & \\
\hline Female & 11 & 0 & 1 & 6 & 4 & \\
\hline Age (years) & & & & & & $\mathrm{P}>0.05$ \\
\hline$<50$ & 47 & 0 & 2 & 27 & 18 & \\
\hline$\geq 50$ & 72 & 0 & 4 & 46 & 22 & \\
\hline Histological differentiation & & & & & & $\mathrm{P}<0.05$ \\
\hline Well & 27 & 0 & 2 & 11 & 14 & \\
\hline Moderate & 74 & 0 & 3 & 54 & 17 & \\
\hline Poor & 18 & 0 & 1 & 8 & 9 & \\
\hline \multicolumn{7}{|l|}{ Hepatitis viral infection } \\
\hline HBV- & 6 & & & & & \\
\hline $\mathrm{HBV}+$ & 113 & & & & & \\
\hline \multicolumn{7}{|l|}{ Tumor size $(\mathrm{cm})$} \\
\hline$<5$ & 67 & & & & & \\
\hline$\geq 5$ & 52 & & & & & \\
\hline TNM staging & & & & & & $\mathrm{P}>0.05$ \\
\hline $\mathrm{I}$ & 17 & 0 & 1 & 9 & 7 & \\
\hline II & 56 & 0 & 2 & 38 & 16 & \\
\hline III & 42 & 0 & 3 & 25 & 14 & \\
\hline IV & 4 & 0 & 0 & 1 & 3 & \\
\hline \multicolumn{7}{|l|}{$\mathrm{AFP}(\mathrm{ng} / \mathrm{ml})$} \\
\hline$<20$ & 37 & & & & & \\
\hline$\geq 20$ & 82 & & & & & \\
\hline
\end{tabular}


A

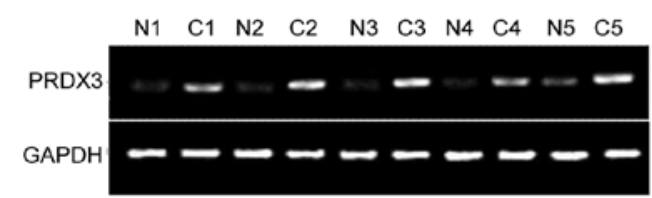

C

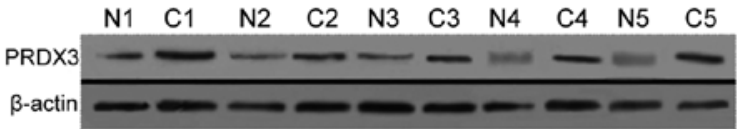

E

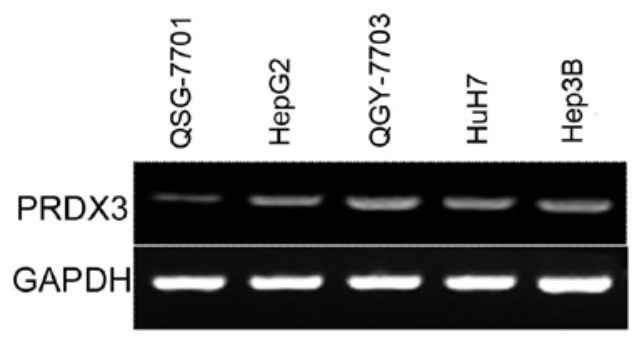

G

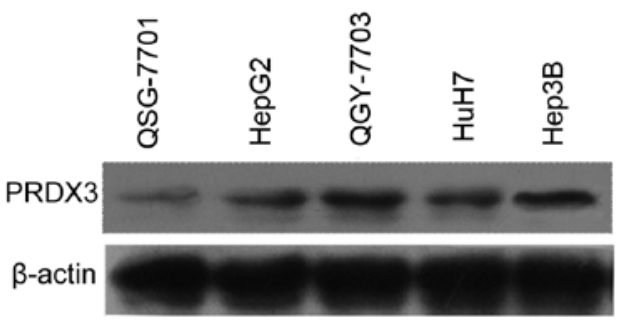

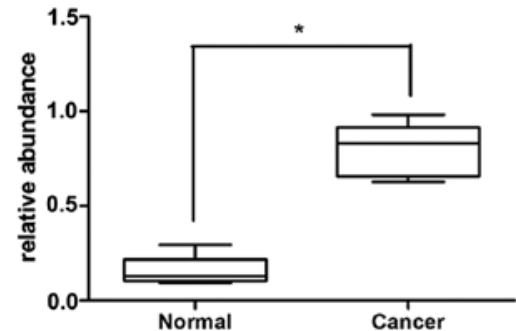

D

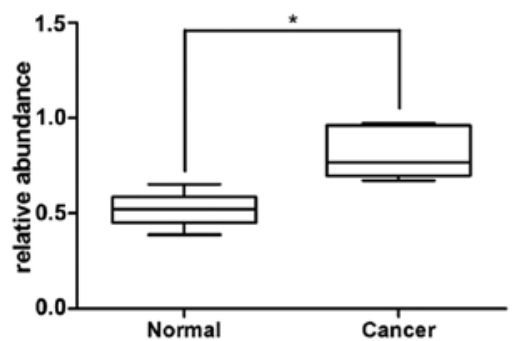

$\mathrm{F}$

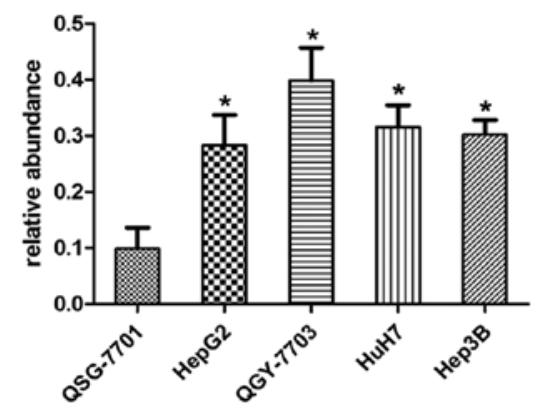

H

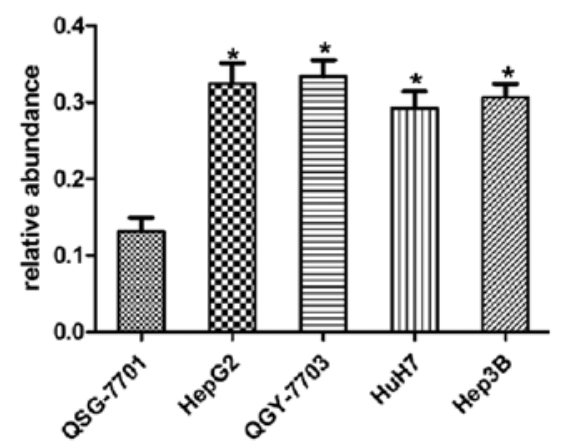

Figure 3. Validation of PRDX3 expression in liver tissues or cell lines. (A) Expression level of PRDX3 mRNA in 40 pairs of HCC tissues and the adjacent noncancerous tissues was identified by RT-PCR. PCR results of 5 pairs are shown in Fig. 3A. (C) Representative results of western blot analysis of paired HCC and the adjacent non-tumor samples with $\beta$-actin as an internal control. (B and D) Box chart, quantitative results of PCR and western blotting of HCC and the corresponding non-tumor tissues. Relative expression levels of PRDX3 mRNA or protein were normalized by GAPDH and $\beta$-actin, respectively. (E) PCR identification results of PRDX3 mRNA expression in liver cancer lines HepG2, QGY-7703, HuH7, Hep3B and the normal liver cell line QSG-7701, and (F) the corresponding expression abundance. (G) Western blot analysis and (H) the quantitative results of the expression of PRDX3 protein in the normal liver cell QSG-7701 and the cancer cell lines HepG2, QGY-7703, HuH7 and Hep3B. "P<0.05, vs. QSG-7701 group, Student's t-test. N, non-tumor tissue. C, cancer tissue.

\section{Discussion}

The mortality rate of HCC has remained relatively high for many years, and its efficacy is limited (24). Therefore, identification of novel tumor biomarkers with higher specificity and sensitivity for the treatment of HCC has the potential to improve the clinical strategy and outcome of HCC.

In the present study, to identify potential biomarkers, comparative proteomic profiling analysis on matched tumor and nontumor tissues identified 22 differentially expressed proteins. Among these, 15 were upregulated, whereas the other 7 were downregulated in HCC. They were classified into several functional groups including metabolism, signal transduction, anti-apoptosis, and redox regulation. (Table I). These proteins could be functionally interrelated to important cellular events, such as cell energy metabolism, antioxidation, cell proliferation, which have been related to the tumorigenesis and progression of malignancies.

PRDX3 belongs to the peroxiredoxin protein family (Prx), which was identified as a cellular defense system against oxidative stress and whose catalytic activity and protein sequences are different from that of other antioxidants (15). As a mitochondrial protein and a c-Myc target gene, PRDX3 catalyzes the reduction of peroxides in the presence of thioredoxin, which is required for mitochondrial homeostasis and neoplastic transformation (17). PRDX3 is an important cellular 


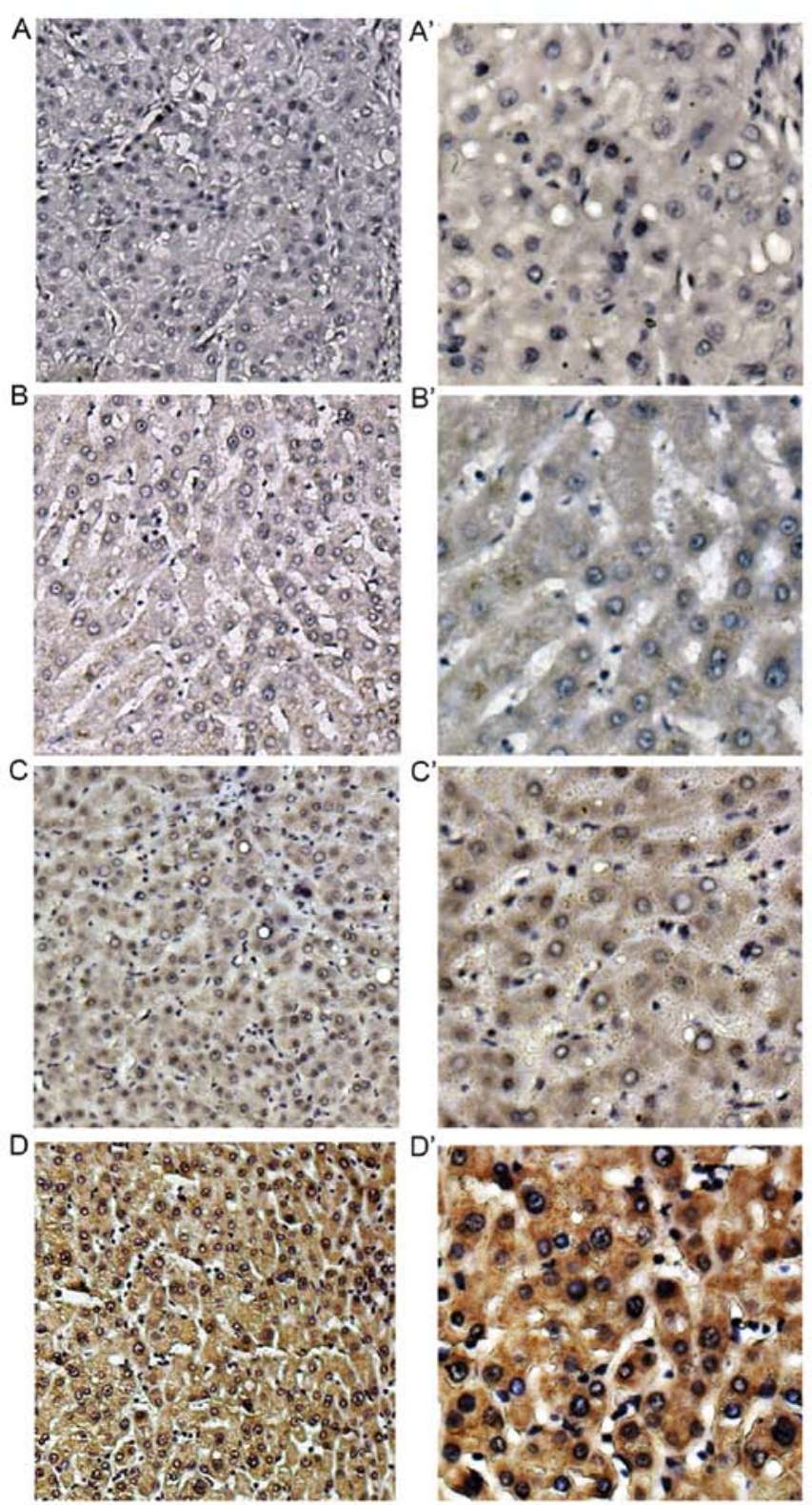

Figure 4. Immunohistochemical analysis of PRDX3 expression in HCC tissues. The expression of PRDX3 was evaluated by immunohistochemistry in $119 \mathrm{HCC}$ specimens and 36 adjacent non-tumor/normal liver tissues. (A) normal liver tissue; (B) well-differentiated; (C) moderately differentiated and (D) poorly differentiated tissues (A-D), original magnification, x200. $\left(A^{\prime}-D^{\prime}\right)$ Representative areas $(A-D)$ at a higher magnification $(x 400)$.

antioxidant that regulates physiological levels of $\mathrm{H}_{2} \mathrm{O}_{2}$ stress to protect cells against apoptosis induced by high levels of $\mathrm{H}_{2} \mathrm{O}_{2}$ (25). Basu et al (26) have shown that PRDX3 is overexpressed in prostate cancer and could be a potential biological determinant of health disparities of patients and novel therapeutic targets. Furthermore, downregulation of PRDX3 could be a possible apoptotic pathway for PP2-induced apoptosis in breast cancer of MCF-7 cells (27). In addition, the overexpression of PRDX3 had also been found in cervical cancer, colorectal cancer and ovarian serous cystadenocarinoma. $(25,28,29)$. However, there are few reports on the relationship between PRDX3 and the progression of HCC, and thus, we focused on the study of thioredoxin-dependent peroxide reductase PRDX3. PRDX3 was found to be upregulated more than 3-fold in HCC tissues, and its differential expression was further verified by RT-PCR and western blotting in a panel of HCC tissues. From our study, we observed that expression of PRDX3 in HCC tissues was higher than that in non-tumorigenic tissues, which was consistent with the results of cell lines (Fig. 3). A higher expression of PRDX3 was observed in metastatic HCC tissues, indicating that PRDX3 may be an important molecule in the development of HCC.

To further study the potential role of PRDX3 as a biomarker for tumor progression, we examined the correlations between PRDX3 expression and HCC staging and histodifferentiation. Our clinicopathological analysis indicated that overexpression of PRDX3 was associated with $94.9 \% \mathrm{HCC}$, and strongly correlated with poor differentiation $(\mathrm{P}<0.05)$. Our studies suggested that PRDX3 has the potential to be developed as a useful biomarker for the therapy of HCC.

In the induction and progression of $\mathrm{HCC}$, reactive oxygen species (ROS) play a critical role (30). Cancer cells are known to have higher levels of intracellular ROS because of increased cellular respiration. High levels of free radicals are thought to have the potential to oxidize macromolecules and thereby to induce mutation of DNA, impairment of protein function, and lipid peroxidation, which lead to tumor development (31). Several studies have shown that tumor cells become more resistant to hypoxia or oxidative stress-induced cell death during carcinogenesis (32).

Increased mitochondrial ROS generation and the disturbance of peroxiredoxin (Prx) production in cancer cells may lead to oxidative stress and hypoxic microenvironments, and subsequently to the induction of apoptosis (17). The upregulation of antioxidant enzymes, especially in mitochondria, would provide the tumor cells with certain survival advantages under their intrinsically high oxidative conditions (31). To protect against oxidative stress induced by ROS, cells use several antioxidants or reductants to maintain the intracellular redox environment in a highly reduced state (33). Therefore, increased expression levels of PRDX3 were assumed to protect tumor cells against the hypoxia microenvironment and drug-induced hydrogen peroxide-dependent apoptosis. Data presented in this study suggest that malignant cells are usually under intrinsic oxidative stress and produce high levels of ROS. Thus, inhibiting mitochondrial antioxidant enzymes in malignant cells may act as an effective cancer therapeutic method.

In conclusion, we analyzed the comparative proteomic profiles between HCC tumors and adjacent non-tumor tissues by 2 -DE and MALDI-TOF MS, and identified differentially expressed molecules which may help to develop effective therapeutic strategies against HCC. PRDX3 was found and confirmed to predict the poor differentiation of HCC based on proteomics screening and molecular biology confirmation. The results of our study suggest that PRDX3 has substantial clinical impact, but more extensive investigations on the role of PRDX3 in the progression of HCC are warranted.

\section{Acknowledgements}

This investigation was supported by Fundamental Research Funds for the Central Universities Fund (303273442, 303275899, 303274073, 303274777) and Key Science and Technology Development Program of Wuhan (201161038344-01). 


\section{References}

1. Avila MA, Berasain C, Sangro B and Prieto J: New therapies for hepatocellular carcinoma. Oncogene 25: 3866-3884, 2006.

2. Yoo HY, Patt CH, Geschwind JF and Thuluvath PJ: The outcome of liver transplantation in patients with hepatocellular carcinoma in the United States between 1988 and 2001: 5-year survival has improved significantly with time. J Clin Oncol 21: 4329-4335, 2003.

3. Llovet JM, Schwartz M and Mazzaferro V: Resection and liver transplantation for hepatocellular carcinoma. Semin Liver Dis 25: $181-200,2005$

4. Frank R and Hargreaves R: Clinical biomarkers in drug discovery and development. Nat Rev Drug Discov 2: 566-580, 2003.

5. Li Z, Zhao X, Bai S, et al: Proteomics identification of cyclophilin a as a potential prognostic factor and therapeutic target in endometrial carcinoma. Mol Cell Proteomics 7: 1810-1823, 2008.

6. Wu FX, Wang Q, Zhang ZM, et al: Identifying serological biomarkers of hepatocellular carcinoma using surface-enhanced laser desorption/ionization-time-of-flight mass spectroscopy. Cancer Lett 279: 163-170, 2009.

7. Hlavata I, Vrana D, Smerhovsky Z, et al: Association between exposure-relevant polymorphisms in CYP1B1, EPHX1, NQO1, GSTM1, GSTP1 and GSTT1 and risk of colorectal cancer in a Czech population. Oncol Rep 24: 1347-1353, 2010.

8. Kanmura S, Uto H, Sato Y, et al: The complement component $\mathrm{C} 3 \mathrm{a}$ fragment is a potential biomarker for hepatitis $\mathrm{C}$ virus-related hepatocellular carcinoma. J Gastroenterol 45: 459-467, 2010.

9. Chuthapisith S, Layfield R, Kerr ID, Hughes C and Eremin O: Proteomic profiling of MCF-7 breast cancer cells with chemoresistance to different types of anti-cancer drugs. Int J Oncol 30: 1545-1551, 2007.

10. Jo M, Nishikawa T, Nakajima T, et al: Oxidative stress is closely associated with tumor angiogenesis of hepatocellular carcinoma. J Gastroenterol 46: 809-821, 2011.

11. Trachootham D, Alexandre J and Huang P: Targeting cancer cells by ROS-mediated mechanisms: a radical therapeutic approach? Nat Rev Drug Discov 8: 579-591, 2009.

12. Oberley LW and Buettner GR: Role of superoxide dismutase in cancer: a review. Cancer Res 39: 1141-1149, 1979.

13. McIlwain CC, Townsend DM and Tew KD: Glutathione S-transferase polymorphisms: cancer incidence and therapy. Oncogene 25: 1639-1648, 2006.

14. Hu YJ and Diamond AM: Role of glutathione peroxidase 1 in breast cancer: loss of heterozygosity and allelic differences in the response to selenium. Cancer Res 63: 3347-3351, 2003.

15. Csiki I, Yanagisawa K, Haruki N, et al: Thioredoxin-1 modulates transcription of cyclooxygenase-2 via hypoxia-inducible factor1alpha in non-small cell lung cancer. Cancer Res 66: 143-150, 2006.

16. Dietz KJ, Horling F, Konig J and Baier M: The function of the chloroplast 2-cysteine peroxiredoxin in peroxide detoxification and its regulation. J Exp Bot 53: 1321-1329, 2002.
17. Song IS, Kim HK, Jeong SH, et al: Mitochondrial peroxiredoxin III is a potential target for cancer therapy. Int J Mol Sci 12: 7163-7185, 2011.

18. Wonsey DR, Zeller KI and Dang CV: The c-Myc target gene PRDX3 is required for mitochondrial homeostasis and neoplastic transformation. Proc Natl Acad Sci USA 99: 6649-6654, 2002.

19. Rabilloud T, Heller M, Gasnier F, et al: Proteomics analysis of cellular response to oxidative stress. Evidence for in vivo overoxidation of peroxiredoxins at their active site. J Biol Chem 277: 19396-19401, 2002.

20. Noh DY, Ahn SJ, Lee RA, Kim SW, Park IA and Chae HZ: Overexpression of peroxiredoxin in human breast cancer. Anticancer Res 21: 2085-2090, 2001.

21. Choi JH, Kim TN, Kim S, et al: Overexpression of mitochondrial thioredoxin reductase and peroxiredoxin III in hepatocellular carcinomas. Anticancer Res 22: 3331-3335, 2002.

22. Kinnula VL, Lehtonen S, Sormunen R, et al: Overexpression of peroxiredoxins I, II, III, V, and VI in malignant mesothelioma. J Pathol 196: 316-323, 2002.

23. Dai Z, Yin J, He H, et al: Mitochondrial comparative proteomics of human ovarian cancer cells and their platinum-resistant sublines. Proteomics 10: 3789-3799, 2010.

24. Camma C, Schepis F, Orlando A, et al: Transarterial chemoembolization for unresectable hepatocellular carcinoma: meta-analysis of randomized controlled trials. Radiology 224: 47-54, 2002.

25. Li XQ, Zhang SL, Cai Z, Zhou Y, Ye TM and Chiu JF: Proteomic identification of tumor-associated protein in ovarian serous cystadenocarcinoma. Cancer Lett 275: 109-116, 2009.

26. Basu A, Banerjee H, Rojas H, et al: Differential expression of peroxiredoxins in prostate cancer: consistent upregulation of PRDX3 and PRDX4. Prostate 71: 755-765, 2011.

27. Liu X, Feng R and Du L: The role of enoyl-CoA hydratase short chain 1 and peroxiredoxin 3 in PP2-induced apoptosis in human breast cancer MCF-7 cells. FEBS Lett 584: 3185-3192, 2010.

28. Kim K, Yu M, Han S, et al: Expression of human peroxiredoxin isoforms in response to cervical carcinogenesis. Oncol Rep 21: 1391-1396, 2009.

29. Wu XY, Fu ZX and Wang XH: Peroxiredoxins in colorectal neoplasms. Histol Histopathol 25: 1297-1303, 2010.

30. Marra M, Sordelli IM, Lombardi A, et al: Molecular targets and oxidative stress biomarkers in hepatocellular carcinoma: an overview. J Transl Med 9: 171, 2011.

31. Chang TS, Cho CS, Park S, Yu S, Kang SW and Rhee SG: Peroxiredoxin III, a mitochondrion-specific peroxidase, regulates apoptotic signaling by mitochondria. J Biol Chem 279: 41975-41984, 2004

32. Ryter SW, Kim HP, Hoetzel A, et al: Mechanisms of cell death in oxidative stress. Antioxid Redox Signal 9: 49-89, 2007.

33. Spitz DR, Azzam EI, Li JJ and Gius D: Metabolic oxidation/ reduction reactions and cellular responses to ionizing radiation: a unifying concept in stress response biology. Cancer Metastasis Rev 23: 311-322, 2004. 